\title{
Persuasive Applications for the Healthy Lifestyle
}

\author{
Md Sanaul Haque \\ Research Unit of Medical Imaging, \\ Physics and Technology (MIPT) \\ Faculty of Medicine \\ INTERACT Research Unit \\ Faculty of ITEE \\ University of Oulu \\ Oulu, Finland \\ md.haque@oulu.fi
}

Permission to make digital or hard copies of all or part of this work for personal or classroom use is granted without fee provided that copies are not made or distributed for profit or commercial advantage and that copies bear this notice and the full citation on the first page. Copyrights for components of this work owned by others than ACM must be honored. For all other uses, contact the Owner/Author.

MUM 2017, November 26-29, 2017, Stuttgart, Germany C 2017 Copyright is held by the owner/author(s).

ACM ISBN 978-1-4503-5378-6/17/11.

http://doi.org/10.1145/3152832.315

\begin{abstract}
People spend a large portion of time at their workplace and henceforth, need actions to recharge their mind and body. Therefore, it is important to highlight on health promotion activities in the workplace that focus on changing personal health behaviours such as diet. Research has revealed that physical activities increase work efficiency [1] and planned naps taken during work keep suitable levels of waking function for day-time and night-time work [2]. Most relevant research has been done without considering the theoretical concept and empirical approach. This study aims to develop persuasive applications incorporating psychological theories to combine these implicit measures such as consuming a healthy diet, physical activity and napping at the workplace. The quantitative and qualitative approach will be used to empirically measuring the effectiveness of the applications in the context of employees' health behaviour change. It is hoped that users will benefit from improved health using the applications.
\end{abstract}

\section{Author Keywords}

Persuasive applications; psychological theories, usercentered design, empirical analysis. 


\section{ACM Classification Keywords}

H.5.m. HCI, persuasive technology.

\section{Introduction}

People typically follow the very same daily procedures and routines, waking up in the morning, going to the workplace, spend a large part of the time in work, and finally returning to his/her nest with an exhausted body and mind. The risk of chronic/long-term diseases such as diabetes, heart disease and cancer can be caused by unhealthy diets [3]. Change of typical physiologic processes is connected with the physical inactivity and the processes can result in changed energy balance, muscle atrophy, and reduced exercise capacity etc. ${ }^{1}$. Mental health problems, stroke, type 2 diabetes and the risk of obesity can be caused by physical inactivity. A number of long-term diseases are caused by these perils which lead to the reasons for death [4]. People might be affected by these perils if they do not retain the healthy diet and physical activity in their working set. Moreover, WHO has acknowledged the workplace as a priority-setting in encouraging health and wellbeing [5]. Workplace dietary interventions are generally effective [6]. Exercise and Healthy diet can increase employees' efficiency at the workplace setting ${ }^{2}$. However, people can feel lazy to do a task willingly but may be deceived by external triggers e.g. reminders i.e. text messaging had been applied on diabetic patients to progress in their self-efficacy to control glycemic [7]. In recent times, persuasive applications have been recognised widely to focus on

${ }_{1}^{1}$ World Health Organization. 2016. Global Activity on Diet, Physical activity and health. http:www.who.int/mediacentre /news/releases/release23/en/ the behavioural change [8]. Supporting behavioural changes become more effective under persuasion [9]. The persuasive application is referred to the interactive application that is intended to change individuals' attitudes and behaviours [10]. The persuasive application has been suggested as an actual technique to boost behaviour change and it has a potential for improving health-related quality of life [10]. More than approximately, 40,000 smartphone-based Apps are available to persuade the users to change their health beahviour e.g. physical activity [11]. Research has highlighted to evaluate the effectiveness of health applications [12]. Nevertheless, there is a number of research articles exist in this extent [13], the content analysis of the mobile phone Apps revealed the use of behavioural change theories or persuasive application and encloses diminutive evidence-based content [14, $15,16]$. Furthermore, studies where behavior change theories in applications were used have concluded that health and wellbeing application lacks the use of theoretical constructs $[17,18]$. It is, therefore, necessary to make the better use of evidence and theory in designing the mobile application dedicated for the users to encourage their behaviour change [19, 20]. Research has confirmed diminutive evidence on the theoretical methodologies to crafting the persuasive applications [21, 22] and diminutive empirical evidence on persuasive applications [13] in enabling healthy lifestyle actions on employees while designing the application followed by the theoretical concepts.

2 Huffington post. 2012. Health workplace productivity eating nutrition exercise. http://www.huffingtonpost.com/ 2012/08/12/health-workplace-productivity-eating-nutritionexercise n 1752749.html? 


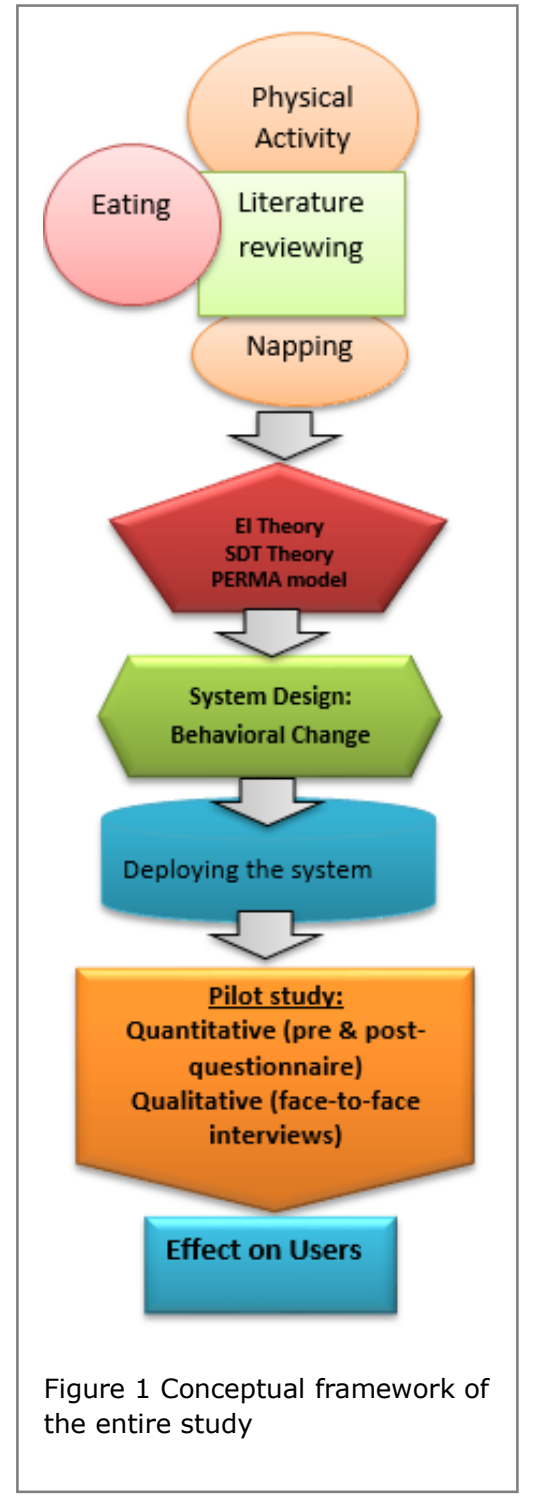

Gethealth ${ }^{3}$ is an in-built mobile application for employees and an online dashboard for employers allowing for active monitoring and management of their wellness plans. Employees use the application to track their daily health tasks (move, munch and mind). The move is to do physical activity, munch is to eat healthily, and mind is to keep one's mind peaceful i.e. napping. Moreover, the application gives the employees to earn rewards (e.g. points) i.e. used the gamified method. However, we did not see whether the Gethealth application used scientific measurements or any empirical approach. This study meditates theoretical constructs, applies scientific measurements while designing, i.e. the User-Centered Design (UCD) process, and implements the applications empirically. The purpose of the study is to deepen understanding of the actual pursuance of the persuasive application on employees in a sense of their healthy lifestyle actions in the workplace such as office environment.

\section{Objectives and Research Question}

This study aims firstly to develop a novel persuasive health application incorporating state-of-the-art theories for the assessment of eating healthy food, physical activity and napping at the workplace. Secondly, the study will utilize the persuasive application to measure its effect in a sense of employees' healthy lifestyle actions needed for the working environment. The main objectives of the study are to explore the effect of persuasive applications on a sense of assessment and measurement of the healthy lifestyle in the context of employees' workplace productivity. The other objectives are to map the state

${ }^{3}$ Gethealthapp. 2017. The complete wellness platform. http://gethealthapp.com. of the art in the areas around eating, physical activity and napping behavioural, other related areas and use this to inform the design of prototype health and wellbeing applications; design, build, and deploy the prototype health and wellbeing applications for employees; and investigate the effectiveness of the health and wellbeing applications by the employees of several organizations. The main focus question of the study is:

What is the actual pursuance of the persuasive applications on employees' healthy lifestyle actions at their working setting?

\section{Proposed Research Approach and Methods}

To answer the research question, we shall design and develop applications for assessing the healthier lifestyle i.e. eating, physical activity and napping behaviour change. The design of the applications will be followed by a User-Centered Design (UCD) process to visualize the health behaviour change among the employees. Before designing the applications, a literature review is conducted mostly on established theories on eating behaviour, physical activity and napping, related works and some possible methods to look at (see Figure 1). Possible psychological theories are selected (EI theory, SDT theory and PERMA model) to build the persuasive applications on eating, physical activity and napping behaviour change. Each of these applications will be validated empirically i.e. a pilot study shall be carried out on a group of healthy adults for one-four week. The pilot study will be divided into experimental and control condition. On the experimental condition, participants 
use our designed persuasive application. Participants also use a paper instructions-based/simple application on the control condition. The key measures of the participants (including participants' progress of doing diet, physical activity and napping) will be measured at a daily interval over weekly periods, and issues. The data of suing the applications will be retrieved from the data server built into the applications. Hybrid (quantitative and qualitative) approach will be used to accomplish the goal of this study. To analyze participants' data quantitatively, participants will be provided pre-questionnaire before using the applications and post questionnaire after using the applications. For qualitative information, face-to-face interviews will be conducted with participants. After investigating the effectiveness of these health and wellbeing applications, a technique may be proposed to measure the healthier lifestyle actions on employees at their office environment.

\section{Expected Results}

The workplace benefits: it is hoped that the persuasive application developed by this study will demonstrate the positive productivity of users i.e. employees at the workplace environment which will increase the working hour efficiency and individual users/employees will benefit from improved health. Therefore, the outcome of the study could bring new health and wellbeing application that might propose a technique of how to measure the healthy lifestyle actions on employees needed for their working environment. In future, individual or organization could apply the technique based on persuasive application to boost one's productivity for a balanced healthy lifestyle.

\section{Context of the study}

This study will be carried out in four phases. Firstly, we will conduct the State of the Art of current methods and theoretical framework to engage people in healthy eating, physical activity and napping at the workplace. Secondly, we will design and develop the persuasive applications focusing on theoretical concepts. Thirdly, the pilot study will be conducted to validate the application through an empirical approach. Lastly, necessary courses, peer-reviewed publications and a thesis will be prepared. So far, a literature review is conducted on behavioural change (eating - EI theory, physical activity - SDT and napping - PERMA model). These include a comprehensive review of existing applications which will be used to indicate the design of the persuasive health and wellbeing application. The health and wellbeing applications will be designed using UCD process and will be followed by a usability test with a small number of users to identify and address usability issues. The necessary materials will be developed to run the study, including identifying the procedure, sampling and informed consent etc. The data from the questionnaires will be compared and analyzed statistically using ANOVA technique to draw conclusions on the effect the persuasive applications have on the employee's healthier lifestyle actions and method to measure the healthy lifestyle. After running pilot study, issues that arise can inform changes in the study. Lastly, peer-reviewed publications and thesis shall be prepared as well as all the courses required to meet up the study.

\section{Status of Current Study}

The persuasive application is workable for the users in the long run and to co-create value for health and wellbeing applications, four key value propositions are 
proposed in this current study [23]. These value propositions could bring advantage to the health application to make application supportable for users in the run. We have been able to incorporate psychological theories using UCD to develop two persuasive applications. Our built persuasive applications on eating behaviour [24] and physical activity change [25] are focused on these key value propositions. The persuasive application on eating behaviour significantly reduced unhealthy eating and users felt more successful in improving their eating habits [24]. The physical activity persuasive application, on the other hand, was designed using UCD [26] and evaluated over a week to which users found it useful to do physical activity [25] and based on the result, a four-week pilot study will be conducted and analyzed statistically. Further study will aim at designing the application on napping using the appropriate psychological construct such as PERMA model. It is expected that the completion date of this study is at the end of 2019 .

\section{Acknowledgements}

We thank all the participants who participated in our pilot study and promised to participate for future pilot study.

\section{References}

1. Roland Croce and Michael Horvat. 1992. Effects of reinforcement based exercise on fitness and work productivity in adults with mental retardation. Adapted Physical Activity Quarterly, 9, pp. 148 178.

2. Masaya Takahashi. 2012. Journal of Physiological Anthropology, An official journal of the Japan Society of Physiological Anthropology, 31(6).
3. American Institute for Cancer Research. 2007. Food, nutrition and the prevention of cancer: A global perspective. Washington, DC: World Cancer Research Fund, American Institute for Cancer Research.

4. Eurobarometer. 2010. Sport and Physical Activity. Special Eurobarometer, 334/Wave 72.3.

5. World Health Organization (WHO) report. 2001. Workers health Global Plan of Action, Sixtieth World Health Assembly.

6. Lisa Quintiliani, Signe Poulsen and Glorian Sorensen. 2010. Healthy Eating Strategies in the Workplace. International Journal of Workplace Health Management, 3(3), pp. 182-196. http://doi.org/10.1108/17538351011078929.

7. Victoria Franklin, Annalu Waller, Claudia Pagliari and Stephen Greene. 2006. A randomized controlled trial of Sweet Talk, a text-messaging system to support young people with diabetes, 23(12), pp. 1332-1338.

8. Samir Chatterjee and Alan Price. 2009. Healthy Living with Persuasive Technologies: Framework, Issues, and Challenges. Journal of the American Medical Informatics Association. 16, pp. 171-178.

9. Daniel J. O'Keefe. 2002. Persuasion theory and research (2nd. Sage Publications), Thousand Oaks.

10. BJ Fogg. 2003. Persuasive Technology: Using Computers to Change What We Think and Do. San Fransisco: Morgan Kaufmann.

11. Maged N. Kamel Boulos, Ann C. Brewer, Chante Karimkhani, David B. Buller and Robert P.

Dellavalle. 2014. Mobile medical and health apps: state of the art, concerns, regulatory control and certification. Journal of Public Health Inform, 5(3), doi: 10.5210/ojphi.v5i3.4814.

12. David E. Conroy, Chih-Hsiang Yang and Jaclyn $P$. Maher. 2014. Behaviour change techniques in top 
ranked mobile apps for physical activity, Am J Prev Med, 46(6), pp.649-52.

13. Juho Hamari, Jonna Koivisto and Tuomas Pakkanen. 2014. Do persuasive technologies persuade? a review of empirical studies. Proceedings of the 9th International Persuasive Conference.

14. Logan T. Cowan, Sarah A. Van Wagenen, Brittany A. Brown, Riley J. Hedin, Yukiko Seino-Stephan, P. Cougar Hall and Joshua H. West. 2013. Apps of steel: are exercise apps providing consumers with realistic expectations? A content analysis of exercise apps for presence of behavior change theory. Health Educ Behav, 40(2), pp. 133-139.

15. Kristen Azar, Lenard Lesser, Brian Y. Laing, Janna Stephens, Magi S. Aurora, Lora E. Burke and Latha Palaniappan. 2013. Mobile applications for weight management: theory-based content analysis. Am J Prev Med, 45(5), pp. 583-589.

16. John Matthews, Khin Than Win, Harri OinasKukkonen and Mark Freeman. 2016. Persuasive technology in mobile applications promoting physical activity: a systematic review. J Med Syst, 40(3), doi: 10.1007/s10916-015-0425-x.

17. Emily R. Breton, Bernard F. Fuemmeler and Lorien C. Abroms. 2011. Weight loss-there is an app for that! But does it adhere to evidence-informed practices? Transl Behav Med, 1, pp.523-529.

18. Joshua H. West, P. Cougar Hall, Carl L. Hanson, Michael D. Barnes, Christophe Giraud-Carrier and James Barrett. 2012. There's An App for That: Content Analysis of Paid Health \& Fitness Apps. Journal of Medical Internet Research. 14(3):e72.

19. Lorien C. Abroms, Nalini Padmanabhan, Lalida Thaweethai and Todd Phillips. 2011. iPhone apps for smoking cessation: a content analysis. Am J Prev Med, 40(3), pp. 279-285.

20. Carolyn Rabin and Beth Bock. 2011. Desired features of smartphone applications promoting physical activity. Telemed J E Health, 17(10), pp. 801-803, doi:10.1089/tmj.2011.0055

21. Saskia M. Kelders, Robin N. Kok, Hans C. Ossebaard and Julia E. Van Gemert-Pijnen. 2012 Persuasive system design does matter: a systematic review of adherence to web-based interventions. J Med Internet Res, 14(6).

22. Oscar E. Pinzon, O. and Sriram Iyengar. 2012. Persuasive technology and mobile health: a systematic review. 7th International Persuasive Conference, Linköping, Sweden.

23. Md S. Haque, Arefeen Arman, Maarit Kangas, Timo Jämsä and Minna Isomursu. 2017. Towards Value Propositions for Persuasive Health and Wellbeing Applications. ICBHI 2017, Proceedings of International Federation for Medical and Biological Engineering. November 2017, Thessaloniki, Greece. [In-Press].

24. Anne Hsu, Jing Yang, Yigit Yilmaz, Md S. Haque, Cengiz Can and Ann Blandford. 2014. Persuasive technology for overcoming food cravings and improving snack choices. Proceedings of ACM CHI, 2014, Canada.

25. Md S. Haque, Wali M. Abdullah, Sadiqur Rahman, Maarit Kangas and Timo Jämsä. 2016. Persuasive health and wellbeing application: A theory-driven design in promoting physical activity. Proceedings of IEEE MEDITEC 2016, Dhaka, Bangladesh.

26. Md S. Haque, Maarit Kangas and Timo Jämsä. 2017. A theory-driven system model to promote physical activity in the working environment with a persuasive and gamified application. Proceedings of DDGD Workshop 2017, Tampere, Finland. 